patients with myelitis but not in any of the opticus neuritis cases. NMOSD seems to be a fairly common cause of SLE associated myelitis, accounting for $60 \%$ of SLE cases with myelitis in our study. SLE patients with engagement of the spinal cord or optic nerve should be screened for anti-aquaporin-4 antibodies. This is important since rituximab rather than cyclophosphamide is the preferred treatment for NMOSD

\section{FACTORS INFLUENCING ON HEALTH-RELATED QUALITY OF LIFE IN FEMALE SYSTEMIC LUPUS ERYTHEMATOSUS PATIENTS WITH FIBROMYALGIA}

${ }^{1} \mathrm{M}$ Su-Jin*, ${ }^{2}$ YS Hong. 'Bucheon St.Mary's Hospital - The Catholic University of Korea, Division of Rheumatology- Department of Internal Medicine, Bucheon, Republic of Korea; ${ }^{2}$ Incheon St. Mary's Hospital, Division of Rheumatology- Department of Internal MedicineCollege of Medicine- The Catholic University of Korea, Incheon, Republic of Korea

\subsection{6/lupus-2017-000215.196}

Background and aims Health-related quality of life (HRQoL) among systemic lupus erythematosus (SLE) patients is reduced, and fibromyalgia contribute to the decreased HRQoL. The objective of the present study is to evaluate the contributing factors for reduced HRQoL in female SLE patients regarding the presence of fibromyalgia.

Methods The HRQoL measurement was made using the SF-36 and Euroqol EQ-5D. Sleep quality, fatigue severity, fibromyalgia severity, and SLE disease associated variables were measured.

Results The scores of HRQoL, including overall scores as well as the physical component summary (PCS) and mental component summary (MCS), were lower in female SLE patients with fibromyalgia $(n=41)$, than in those without fibromyalgia $(n=111)$. SLE patients with fibromyalgia showed higher SLE disease activity, and more severe fatigue score, depressive mood and deteriorated sleep quality, compared with patients without fibromyalgia. In SLE patients with fibromyalgia, education level, SLE organ damage, fatigue severity, sleep quality and fibromyalgia severity were significantly correlated with EQ-5D, whereas age, income, SLE disease activity, steroid dose, and disease duration were not correlated with EQ-5D. On the other hand, education level did not show significant correlation with EQ-5D in SLE patients without fibromyalgia. Multivariate logistic regression analysis revealed that depressive mood is only independent contributing factors for deteriorated HRQoL in female SLE patients with fibromyalgia. Interestingly, in SLE patients without fibromyalgia also showed same result.

Conclusions The quality of life in SLE patients can be improved by managing depressive mood both in patients with fibromyalgia and in those without fibromyalgia.

\section{A RETROSPECTIVE ANALYSIS ON SYSTEMIC LUPUS ERYTHEMATOSUS IN THE INDIGENOUS AND NON- INDIGENOUS POPULATION IN CENTRAL AUSTRALIA FOCUSSING ON TREATMENT AND OUTCOMES OF LUPUS NEPHRITIS}

${ }^{1} \mathrm{P}$ Subramani*, ${ }^{2} \mathrm{~S}$ Brady, ${ }^{1} \mathrm{~S}$ Thomas, ${ }^{1} \mathrm{~B}$ Pawar. 'Alice Springs Hospital, Dept. of Nephrology, Northern Territory, Australia; ${ }^{2}$ Alice Springs Hospital, Dept. of Medicine, Northern Territory, Australia

10.1136/lupus-2017-000215.197
Aim To analyse and compare the prevalence, manifestations and outcomes of systemic lupus erythematosus (SLE) in the Indigenous and non-Indigenous population in Central Australia.

Background SLE is a common autoimmune condition worldwide. With the development of better immunosuppression, outcome of the disease has significantly improved. There is a high prevalence of SLE in the Indigenous population in Central Australia.

Methods The medical records of all patients diagnosed and/or being treated for SLE at Alice Springs Hospital from 1999 to March 2016 were reviewed. Only those with definite SLE, defined by the 2012 Systemic Lupus International Collaborating Clinics (SLICC) were included in this study.

Results 39 patients fulfilled the criteria, 31 were Indigenous. 37 were female. The prevalence of SLE was 1:601 in the Indigenous and 1:4051 in the non-Indigenous. Both the groups fulfilled an average of 6 SLICC criteria. 18 patients 17 of whom were Indigenous, had biopsy proven lupus nephritis. The ISN-RPS 2003 lupus nephritis Class IV and V was most prevalent, followed by Class III. Various immunosuppressive regimes were used to treat lupus nephritis with varying responses. The Indigenous group had a high predisposition to infections, and the risk increased with immunosuppressive therapy. Non-adherence to treatment was a significant problem in the Indigenous group. 5 patients were deceased, 4 of whom were Indigenous.

Conclusions There is a high prevalence of SLE in the Indigenous population in Central Australia. A low threshold for renal biopsy is recommended for classification and treatment purposes. Treatment regimes and response varied between individuals.

\section{TTP SECONDARY TO SLE: RITUXIMAB IMPROVES OVERALL BUT NOT RENAL SURVIVAL}

F Sun*, X Wang, W Wu, K Wang, Z Chen, T Li, S Ye. Ren Ji Hospital South Campus- School of Medicine- Shanghai JiaoTong University, Department of Rheumatology, Shanghai, China

\subsection{6/lupus-2017-000215.198}

Background and aims Thrombotic thrombocytopenic purpura (TTP), a form of thrombotic microangiopathies (TMA), is a series of life-threatening disorders. Systemic lupus erythematosus (SLE) is one of most common acquired causes. To identify predictors of prognosis in patients with TTP secondary to SLE, we conducted a single-centre historical study.

Methods Using the electronic medical record system which includes all clinical data of patients who were hospitalised in the department of Rheumatology in Ren Ji Hospital from 2013 January to 2016 June, we identified patients with the query terms "SLE", "schistocyte", "TTP", and "TMA". Of 2182 SLE patients, a total of 21 consecutive patients with TTP secondary to SLE were enrolled.

Results The 90 day short-term mortality was 33.3\%. The kidney involvement (66.7\%) was associated with poor prognosis, while the administration of rituximab $(n=13)$ was an independent protective factor according to logistic regression analysis. Although compared to conventional treatment, i.e., plasma exchange, high dose glucocorticoids and intravenous immunoglobulin, the overall survival is significantly higher among patients receiving rituximab add-on $(92.2 \%$ vs $25 \%$, 